\title{
Wirtschaftsethische Reflexion ökologischer Bildung in und von Organisationen
}

In der Debatte zu den ökologischen Herausforderungen unserer Zeit werden zuweilen die Umweltschutzaspekte mit einem Bedeutungsüberschuss versehen. Außer Frage steht jedoch: Es müssen Konzepte, Strukturen und Prozesse entwickelt werden, die eine Gestaltung der nachhaltigen Entwicklungen ermöglichen. Von Thomas Prescher

\section{Einleitung}

Es wird immer wieder festgestellt: „So können wir nicht weitermachen. Was wir tun, ist unverantwortlich" (Eppler 2011, S. 28). Die zentralen Fragestellungen im Beitrag sind daher:

I Kann mithilfe wirtschaftsethischer Kommunikation eine Grundlage für das Konzept der nachhaltigen Entwicklung mit einer darauf bezogenen ökologischen Bildung in und von Organisationen geschaffen werden?

I Kann die Pädagogik, insbesondere die Organisationspädagogik, einen Beitrag für ein wirtschaftsethisches Handeln leisten?

I Welcher Begriff der ökologischen Bildung ergibt sich aus der reflexiven Betrachtung über die Wirkungshorizonte im Kontext von Organisationen?

Für diese Fragen ist zunächst eine systemtheoretische Bestimmung des Verhältnisses von Ethik und Wirtschaft (spezieller: Ökonomie) notwendig. Mit dieser Verhältnisbestimmung soll anhand einer Analyse zu Programmen zur Förderung der nachhaltigen Entwicklung in Unternehmen (509 Programme von 179 Anbietern) herausgearbeitet werden, dass für den Kontext ökologischer Bildung und nachhaltiger Entwicklung im Wesentlichen zwischen einer strukturellen Verantwortung durch umweltpolitische Institutionen und einer nicht beobachtbaren moralischen Verantwortung unterschieden werden kann. Damit wird der Analyserahmen von Ökonomie, Ethik und Ökologie im Beitrag geschlossen. Im Sinne des herausgearbeiteten Verständnisses und aus Perspektive der ökologischen Debatte wird das Ergebnis begrifflich und provokativ als "verantwortungslose Verantwortung“ der Unternehmen zugespitzt.

\section{Wirtschaftsethik in der funktional differenzierten Gesellschaft}

Im Diskurs um die nachhaltige Entwicklung scheint es nicht um eine Nachhaltigkeit im eigentlichen Sinne zu gehen, sondern um die Sicherung strategisch wichtiger Optionen. Ethische Kommunikation spielt hierbei eine bedeutsame Rolle, da in der heutigen Zeit die Allokation von Marktchancen durch die Unternehmen zunehmend an Bedeutung gewinnt. Dabei muss die Frage gestellt werden, ob ethische Kommunikation ausschließlich ökonomisch Sinn macht (These: Ethische Kommunikation ist ökonomisch) oder ob durch den Diskurs die Ökonomie auch mehr ethisch sein kann (These: Ökonomie ist ethisch, Karmasin 1996). Aus diesem Grund soll im Folgenden eine funktionale und nicht normative Begründung der Wirtschaftsethik im Zentrum stehen.

Insbesondere unter der systemtheoretischen Annäherungsweise an das Thema treten Schwierigkeiten bei der Verhältnisbestimmung von Moral, Ethik und Wirtschaft auf, da eine moralische Kommunikation mit dem Code Achtung - Missachtung nicht einfach in die Kommunikation der jeweiligen Teilsysteme der Gesellschaft umgeformt und übertragen werden kann (Homann 1995). Der Moral kommt eher die Aufgabe der Irritation von Systemen zu, da die Selbsterhaltung der Systeme nach den jeweiligen Funktionscodes mit ihren Funktionserfordernissen erfolgt und so die Transaktionskosten für das Unternehmen reguliert. Die Beziehung zwischen Ökonomie und Ethik kann daher als Differenz verstanden werden (Wieland 1991).

Prinzipiell lässt sich Ethik dazu als eine theoretische Begründung der Moral definieren, das heißt als wissenschaftliche Disziplin. Moral kann dabei als Kommunikation verstanden werden, die sich mit pathologischen Fällen - Ausnahmefällen wie zum Beispiel ökologisches Fehlverhalten einer Branche oder eines Unternehmens - befasst. Sie ist keine Alltagskommunikation, sondern sie wird gewählt, wenn es notwendig erscheint, der Kommunikation Nachdruck zu verleihen (Luhmann 1989). Es geht dann in der Ethik um eine Begründungsreflexion von Urteilen, Regeln und Prinzipien, die angeben, was denn erwartet werden darf und woran man sich halten kann.

Ein Grundproblem besteht darin, dass sich die Ethik einseitig am Guten orientiert. „Die Ethik könnte man sagen, unternimmt den Versuch, selbst gut zu sein [...] (Luhmann 1990, S. 6). Nur können sich die Regeln sozialer Systeme in einer 
funktional differenzierten Gesellschaft nicht einseitig am $\mathrm{Gu}$ ten orientieren, da sie den Anforderungen der spezifischen Operationsweise dieser Systeme nicht gerecht werden würde. „Unternehmen sind dazu da, Gewinne zu machen, und wenn sie keine Gewinne machen, hören sie auf zu existieren" (Hauff 1991, S. 82). Der Unterschied von ökonomischer Kommunikation zu moralischer Kommunikation besteht darin, dass erstere aus Preissignalen besteht. Die Ökonomie kann also unter theoretischer Betrachtung nicht mithilfe moralischer Kommunikation gesteuert werden, sondern steuert sich in erster Linie nach systeminternen Kriterien: Güterangebote signalisieren Zahlungsnachfrage, Güternachfragen signalisieren Zahlungsangebote (Luhmann 1988).

\section{Von der Individual- und Tugendethik zur Struktur- und Prozessethik}

Die Frage ist nun, warum es eine solche Vielzahl von Publikationen gibt, in denen das Bedürfnis der Wirtschaftsakteure vermutet werden kann, sich verbal mit der Öffentlichkeit zu arrangieren, und ob die Ethik die richtige Adresse ist, die durch das Wirtschaftssystem erzeugten ökologischen Probleme in der modernen Gesellschaft zu adressieren und zu lösen. Eine Moralisierung liegt ja, wie bereits gesagt, dann nahe, wenn Situationen als pathologisch erlebt werden, für die andere verantwortlich sind. Und so mag es verwundern, dass das Thema nicht etwa allein von außen an die Wirtschaft herangetragen wurde, sondern von den großen Firmen selbst als Gegenstand der Unternehmenskommunikation entdeckt wurde (Wieland 1991).

Ethik wird im Kontext von Unternehmen als ein Instrument angesehen, bestehende Steuerungsdefizite zu füllen. Aufgrund einer moralisch geführten Kommunikation fand die nachhaltige Entwicklung eine juristische und ordnungspolitische Würdigung mit arbeitspolitischen, technologischen und ökologischen Entscheidungen (Steinmann et al. 1992). In diesem Sinne lassen sich ethisches und ökonomisches Handeln ineinander integrieren und gegenseitig nutzbar machen (Homann 1995).

$\mathrm{Zu}$ den prinzipiellen Gegenstandsbereichen wirtschaftsethischer Kommunikation gehören die interne Leistungserstellung, das Handeln im externen Markt sowie die Anforderung der Gesellschaft an ein Unternehmen (Wieland 1991). Zahlreiche empirische Studien zeigen hierbei, dass Unternehmensethik verstanden als ethisches Verhalten der Unternehmen und als ethisches Verhalten in und gegenüber den Unternehmen weder rein individualethisch noch sozialethisch erklärbar ist. Es gibt hier offensichtlich ein Wechselverhältnis zwischen dem Mikro-, Makro- und Mesokosmos menschlicher Rationalität und menschlichen Handelns, welches durch das jeweilige Nutzenkalkül geprägt ist (Nethöfel 1997).

„Die Frage nach der Ethik der Unternehmung behandelt eine wesentliche Frage ethischer Ökonomie, nämlich den $\mathrm{Zu}$ sammenhang zwischen Individualethik und Sozialethik [...]. In einer Gesellschaft der organisierten Komplexität ist darüber hinaus sinnvoll, auch eine Ethik eben dieser Organisation als strukturelles Problem der Gesellschaft zu behandeln, denn die verselbstständigten Folgen des Handelns treten dem Handelnden als individuell nicht veränderbare Institutionen bzw. Sachzwänge entgegen“ (Kamarsin 1996, S. 168).

Entscheidend ist immer die Bereitschaft der Mitarbeitenden, sich auch dann an die Regeln zu halten, wenn keine unmittelbare soziale Kontrolle besteht. Durch unternehmensethische Maßnahmen kann hier eine gemeinsame Wertebasis im Unternehmen geschaffen werden, die einen verlässlichen Orientierungsmaßstab bietet und so Vertrauen und Loyalität im Unternehmen und in das Unternehmen erzeugt. Eine dafür erforderliche Wirtschaftsethik muss dabei die Defizite einer Individualethik als Tugendethik des sogenannten Einzelunternehmers korrigieren und vielmehr eine Struktur- und Prozessethik sein (Hengsbach 1991). Steinmann und Gerhard (1992) argumentieren in diesem Zusammenhang für ein dialogisches Prinzip als notwendige Voraussetzung für die Ausgestaltung. Struktur- und Prozessethik meint hier, dass institutionell die Voraussetzungen geschaffen werden, damit innerhalb von Organisationen eine professionelle Zuständigkeit, eine damit zusammenhängende individuenunabhängige Reflexion und Entscheidungsmacht gesichert werden (Krainer et al. 2010).

\section{Aspekte der Wirtschaftlichkeit rangieren vor einer Ethik zur nachhaltigen Entwicklung}

Die Ökologie als Wissenschaft kann als ein umfassendes Deutungsangebot für die durch den Menschen verursachte Umweltkrise verstanden werden, was auf einer traditionell ethisch motivierten Kulturkritik aufbaut (Engels 2006). Die Ökologie wird mit ihren Argumenten immer wieder dazu verwandt, einen Status quo erhalten zu wollen. Gleichzeitig repräsentiert sie aber auch das Bestreben, eine Utopie des Zusammenlebens zu thematisieren, das insbesondere dem Prinzip der Harmonie folgt, wenngleich die dafür nötigen Bedingungen ungeklärt bleiben.

Aufgrund der Popularität, die die Ökologie insbesondere durch ihre Medienwirksamkeit erlangt hat, ist sie mit der Kritik konfrontiert, vor allem gesellschaftliche und ökonomische Aspekte zu vernachlässigen. Kritisiert wird, dass die Lösungen an den alltäglichen Realitäten und den Lebenskonzepten sowie Verhaltensmustern vorbeigehen, weswegen eine ethisch begründete, tatsächliche ökologische Transformation bisher ausbleibt. Diese Lücke soll die Nachhaltigkeitsforschung durch die Entwicklung von Methoden und Konzepten als interdisziplinärer Ansatz schließen.

Die Dimension der Nachhaltigkeit bezieht sich damit nicht mehr nur auf ein Verständnis von Natur als Bezugsphänomen, sondern auf soziale und wirtschaftliche Aspekte. Insbesondere geht es dabei um die Integration von normativen Elementen der jeweiligen Bezugsdisziplin in die tatsächlichen Lebensrealitäten. Towers und Kohler (2008) verstehen Nachhaltigkeit in diesem Sinne als Begriff, der sich historisch nach dem Konzept der Ökologie entwickelt hat. Als zentraler Unterschied wird im 
Nachhaltigkeitskonzept stärker auf den Menschen und sein Handeln verwiesen. Während Ökologie als Wissenschaft dem Gewinnen von Erkenntnis über das Was zu dienen scheint (These: Ökologie als Normensetzung), folgt das Verständnis von Nachhaltigkeit eher der Logik einer Intervention. Einer bestimmten Intervention schreibt man dann eine Wirkung zu. Nachhaltigkeit lässt sich als Element erster Ordnung und damit als nachhaltigkeitsbezogenes Handeln ver-

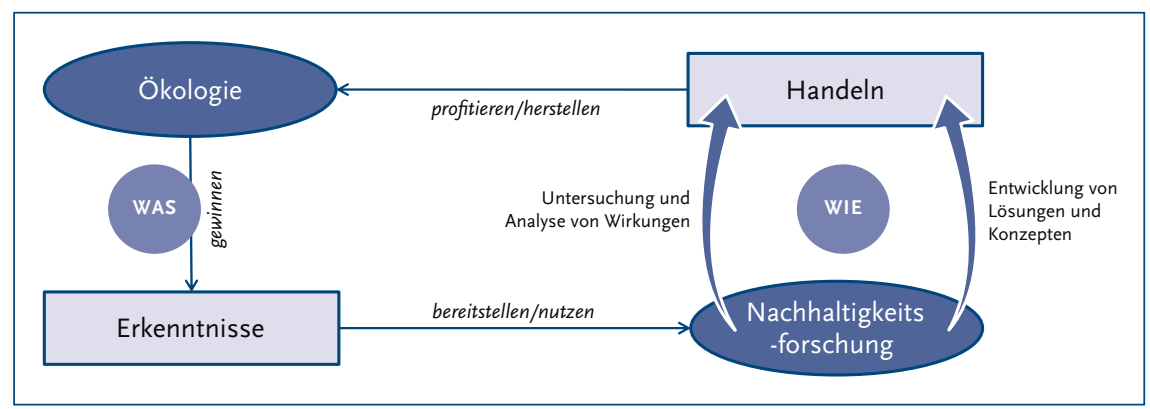

Abbildung 1: Verhältnis Ökologie zur Nachhaltigkeitsforschung stehen. Als Element zweiter Ordnung kann es dagegen als reflexive Beobachtung über gelungenes Handeln gefasst werden (Schüßler 2007).

Nachhaltigkeit bezieht sich auf ein gesellschaftliches Leitbild, welches auf einem Entwicklungsprozess gründet, der im ethischen Sinne sowohl positiv verbessernd als auch dauerhaft angelegt ist. Insofern basiert eine nachhaltigkeitsorientierte Entwicklung auf einer gesellschaftlich anzustrebenden Zielvorstellung, die, anthropozentrisch angelegt, die „[...] Sicherung der menschlichen Existenz, Erhaltung des gesellschaftlichen Produktivkapitals, Bewahrung der Entwicklungs- und Handlungsmöglichkeiten“ (Conrad 2000, S. 6) sichern soll.

Mit Blick auf die ökologische Nachhaltigkeit kann man herausstellen, dass die Nachhaltigkeitsforschung im Unterschied zur Ökologie stärker nach dem Wie der normativen Setzungen fragt (These: Nachhaltige Entwicklung als Normenumsetzung), das heißt, wie das Naturkapital und die Umweltqualität erhalten werden können. Nachhaltigkeitsforschung bezieht sich so gesehen auf das Konzept nachhaltiger Entwicklung (Abbildung 1).

Bezogen auf das dahinterstehende Paradigma des Wachstums als Grundlage der Wohlstandssicherung sehen Beschorner et al. (2005) in der Verwendung des üblichen Nachhaltigkeitsdreiecks als zentrale Norm nachhaltigen Handelns selbst eine Reihe von Missverständnissen und Fehldeutungen über das Verhältnis der Ökonomie zur Ökologie und zum Sozialen. Es erscheint so, als ob man hier auf normativer Ebene versucht, einen Ausgleich zwischen den Elementen dieser Gleichung herzustellen, da in vielen Darstellungen und Diskussionen das ökonomische System mit seiner Markt- und Produktionslogik zwar als normatives Zielsystem erklärt wird, es aber scheinbar einer ethisch vertretbaren Legitimation nach außen bedarf, weiter so zu handeln wie bisher. Beschorner et al. (2005) sprechen hier von einem "naturalistischen Fehlschluss“, weil das „Sein-Sollende“ aus dem „Seienden“ abgeleitet wird. Das heißt, man hält an der Grundlogik der Ökonomie, die gleichzeitig als Ursache der Probleme dieser Welt identifiziert wird, fest und verharrt in der Denkweise der Modernisierung, indem die Probleme offenen Auges erkannt und als beherrschbar bestimmt werden (Eblinghaus et al. 1998).

Im Nachhaltigkeitsdiskurs geht es damit im Wesentlichen um die Frage, wie das bisherige wirtschaftliche Wachstum fortgeschrieben werden kann. Nachhaltigkeit ist demgemäß eine
Kategorie, die lediglich nach der Sozial- und Ökologieverträglichkeit dieser Strategie fragt, ohne grundsätzlich kohärent und inklusiv aufeinander Bezug zu nehmen (Eblinghaus et al. 1998). Ökonomie und Ökologie befinden sich hier in einem Spannungsverhältnis, welches durch eine paradoxe Differenz geprägt ist, die sich, wie es scheint, nicht zu einer Einheit zusammenführen lässt. Aus dieser Paradoxie einer Einheit der Differenz von Ein- und Ausgeschlossenen (Luhmann et al. 1988) ergibt sich eine Spannungslage für Nachhaltigkeit und Ökologie, weil das dominierende ökonomische Prinzip nicht einfach durch eine wie auch immer geartete und ethisch begründete Ökologie ersetzbar ist. Es lässt sich vielmehr schlussfolgern, dass es darauf ankommt, das gegenseitige Verhältnis zwischen Ökonomie und Ökologie „neu zu bestimmen“ und zusammenzuführen. Mit einem sozialwissenschaftlichen Blick betrachtet kann eine ökologische Lebensweise nicht einfach im Spiegel einer „zerstörerischen“ Ökonomie stattfinden, sondern muss eigenständige Operationen und Programme einschließen, die es zu analysieren und zu (re)konstruieren gilt. Eine Einsicht durch Vernunft, auf welche in der ökologischen Debatte häufig gesetzt wird, hat systemtheoretisch in diesem Sinne keine ethische Qualität. Vernunft folgt vielmehr der Verfahrensrationalität des Systems. Vernunft orientiert sich demnach an den Operationen gesellschaftlicher Kommunikation.

Müller-Christ (2008) veranschaulicht diesen Zusammenhang anhand des ökonomischen Wertekontextes als Frame, der auf Wohlstand ausgerichtet ist und der ökonomischen Rationalität von Effizienz folgt. Die Folge ist, dass es der Nachhaltigkeit an einer anschlussfähigen Zielsetzung für die Unternehmen fehlt. Aus diesem Grund, so schlussfolgert der Autor, findet eine Umcodierung des Nachhaltigkeitsframes auf das Effizienzproblem der Wirtschaft statt: Nachhaltigkeit wird zum Instrument der innerbetrieblichen Effizienzsteigerung, weshalb beispielsweise Energieeffizienzmaßnahmen auf technologischer Ebene anschlussfähig sind: „Es interessiert einzig, was sich wie und warum (nicht) rechnet. Weil es auf die Differenz ankommt, tun ökonomisch alle Kosten weh, denn sie schmälern das mögliche Plus“ (Arlt et al. 2015, S. 22). Damit wird der eigentliche Widerspruch zwischen den Wertekontexten und den Notwendigkeiten einer ökologischen Transformation ignoriert.

Diskurstheoretisch lässt sich daher schlussfolgern, dass die Debatte zur Kritik der Ökonomie eine legitimierende Sinnstif- 
tungsfunktion einnimmt (Lederle 2008) und damit eine Basis für die Initiativen schafft, in denen ökologische Bildung als lebensweltlicher Erkenntnisprozess einen Beitrag zur Problemlösung leisten kann.

\section{Bildungstheoretische Standortbestimmung einer ökologischen Bildung von und in Organisationen}

In der Debatte um eine nachhaltige Entwicklung beschreibt man Bildungskonzepte auch als ein funktionales politisches Instrument (Becker 2001), um eine möglichst wirksame Auseinandersetzung mit dem Nachhaltigkeitsleitbild auf der Ebene des Individuums zu gewährleisten. „Die Pädagogisierung eines gesellschaftlichen Problems setzt regelmäßig dann ein, wenn für politisches Handeln kein Ansatz gefunden oder ein Konsens zu erreichen ist" (Radtke 1995, S. 856).

Proske (2002) beschreibt dementsprechend, dass verschiedene gesellschaftliche Problemlagen in pädagogische Probleme und Aufgaben transformiert werden und dass das pädagogische Handeln sich gleichzeitig aus den klassischen Institutionen der Erziehung ablöst. Es wird die Hypothese formuliert, dass eine solche gesellschaftliche Pädagogisierung als ein funktionales Äquivalent zur Steuerung von sozialen Systemen angesehen werden kann - insbesondere dann, wenn eine moralische Kommunikation versagt. Formen pädagogischer Interventionen sollen letztlich die gezielte Beeinflussung von Bewusstsein und Handeln gewährleisten können:

„In diesem Sinne wird in Pädagogisierungsprozessen die Pädagogik zum Platzhalter der Moral in der moralisch dezentrierten Gesellschaft. Mit ihr lassen sich [...] moralische Ansprüche in der Gesellschaft kommunizieren, die ansonsten offenbar über keine Adresse mehr verfügen. In ihrer Absicht, die Gesellschaft durch Erziehung und (Selbst-)Bildung zu verändern, repräsentieren gesellschaftlich entgrenzte pädagogische Kommunikationen den re-entry der in funktional differenzierten Gesellschaften ortlos gewordenen Moral“ (Proske 2002, S. 294).

Ökologische Fragen können vor diesem systemtheoretischen Hintergrund durch Bildung und Erziehung als Fragen an die Funktionssysteme formuliert werden. Ökologische Fragen sind dabei oftmals Inszenierungen von Gefährdungen und bringen zum Ausdruck, wie sich die Gesellschaft zu diesen positioniert. Jedoch führe, so Bolscho (2010), die Thematisierung von Gefährdungen nicht zu einer inneren Betroffenheit im Kontext von Erziehungsprozessen, um daraus veränderte Handlungsalternativen abzuleiten und zu wählen. Der Autor stellt für die Betrachtung erzieherischer und didaktischer Konzepte heraus, dass in der Verortung zu einer sozialökologischen Bildungsforschung Umwelt immer als zentraler Bezugsrahmen herangezogen wird. Auf diese Weise soll das klassische naturwissenschaftliche und sozialökologische Verständnis von Ökologie dazu dienen, in Erziehungsprozessen Diskurse zu entfalten, die zwischen den verschiedenen Funktionssystemen vermitteln. Konzepte der BNE, so de Haan (2008), führen zu einer Dominanz kognitiver Muster. Damit einhergehend wird die Kritik formuliert, dass die Orientierung auf Wissen beziehungsweise Inhalte, die über das Verhältnis zur Umwelt und Natur aufklären, nicht ausreichend sei (Bolscho 2010). Dies erscheint insofern bedeutsam, als die Umweltbewusstseinsforschung deutlich herausstellt, dass eine umweltbezogene Wissensentwicklung nicht zu einem umweltbezogenen Handeln führe (Huber 2011). Die Diskussion über eine ökologische Transformation durch Bildung und Lernen sowie die Kritik am Konzept der BNE für den außerschulischen Bereich (Michelsen et al. 2013) führen daher zu den bildungstheoretischen Fragen, wer lernen sollte und welche Inhalte in kategorialer Hinsicht lernenswert sind. Dies erscheint insofern plausibel, als die Inhalte und Kompetenzansprüche der Gestaltungskompetenz der BNE nicht anschlussfähig erscheinen, da sich darin befindliche Erziehungsziele und Inhalte nicht als Entscheidungsprogramme und Selektionscodes verwenden lassen (Luhmann 2000).

Nachhaltige Bildung muss sich dazu aus formalisierten und organisierten Bildungskontexten lösen und eine systemische Perspektive auf die Prozesse der Systementwicklung in den sozialen Kontexten einnehmen. Konzepte der ökologischen Bildung benötigen einen konkreten Verwertungszusammenhang für die Handlungsebene in den Organisationen. Für ein Bildungsverständnis, das eine Perspektive auf die Gestaltung und Entwicklung von Nachhaltigkeit einnimmt, spielt dazu der „Umgang mit Deutungen“ (Tietgens 1990, S. 57) über die eigene Wirklichkeit eines sozialen Systems und des sozialen Umfelds eine wichtige Rolle. Das Lernen von Nachhaltigkeit lässt sich in Anlehnung an Arnold und Siebert (1995) als ein Deutungslernen und ein lebensweltbezogener Erkenntnisprozess beschreiben. Dabei wird die Perspektive subjektbezogener Deutungen von Lehrkräften und Lernenden verlassen und um die Ebene der Konstruktion des Sozialen im Kontext nachhaltigen Wirtschaftens erweitert. Dabei gerät die Frage des Lernsubjekts neu in den Blick, da nicht nur Individuen, sondern auch Organisationen lernen (Bezug: Wer sollte lernen?). Es geht dabei nicht nur um intentionale Lernprozesse in einem unmittelbaren Subjekt-Subjekt-Verhältnis, sondern auch um funktionale und extensionale Aspekte des Lernens im Rahmen sozialer Institutionen, Strukturen und Prozesse (Treml 2000), damit die Postulate und Handlungsempfehlungen des Leitbildes für nachhaltige Entwicklung als Deutungsentwürfe auf die Alltagspraxis der Funktionäre in Organisationen und Individuen anschlussfähig werden. Es geht konkret um einen organisationspädagogischen Zugriff auf eine ökologische Bildung als Problemlösung.

\section{Programmanalyse als Zugang zur Konstitution ökologischer Bildung in und von Organisationen}

Die sich aus dieser sozialökologischen Systemforschung über das Zusammenspiel von Ökonomie, Ökologie, Nachhaltigkeit, Ethik und Pädagogik ergebenden Erkenntnisse kön- 
nen neue Einsichten über Entwicklungsveränderungen eröffnen. Bedeutsam ist die Perspektive, wie sich die Wirklichkeit einer ökologischen Bildung in und von Organisationen nachzeichnen und explizieren lässt. Ein Ansatz stellt dazu die Methode der Programmanalyse dar. In ihr wird sichtbar, wie sich Programmplanende eine ökologische Bildung für Organisationen vorstellen.

Die Programmanalyse als qualitative und induktiv-deduktive Inhaltsanalyse sowie das Verfahren des topic modeling als Form automatisierter deduktiver Textanalyse dienten als Ansatz der Methodentriangulation. Bei dem Teilprojekt Programme zur Förderung der nachhaltigen Entwicklung in Unternehmen ging es darum, die äußere Seite einer ökologischen Bildung für eine nachhaltige Entwicklung in einem indirekten Zugang in den Blick zu nehmen und die den 505 analysierten Programmen von 179 Bildungsanbietern innewohnenden Bildungsverständnisse zu explizieren. Der Fokus wurde auf die Dimensionen Ökologie und Ökonomie in Bezug auf die Zielsetzung eines wie auch immer gearteten und zu identifizierenden Beitrages zum Umwelt- und Naturschutz gelegt. Im Kern zeigt die Programmanalyse auf, was in der Umsetzung in Unternehmen beziehungsweise auf Seiten der Bildungsanbieter gegenüber den Unternehmen für eine kollektiv geteilte Auslegung der Wirklichkeit vorherrscht. In ihr wird die Konstitution ökologischer Bildung sichtbar, wie sie aus der sozialen Praxis emergiert.

Die Programmanalyse dient dazu, den zu untersuchenden Sachverhalt einer nachhaltigen Entwicklung durch Bildung in und von Organisationen detailliert zu rekonstruieren. Sie wird sich deshalb den Organisationen als Räume für ein Nachhaltigkeitslernen zuwenden, und thematisch adressierte Bildungsangebote für Organisationen und Unternehmen in den Untersuchungsfokus rücken. Um einen differenzierten Zugang zur Konstitution der Angebote für Unternehmen zur Nachhaltigkeit und zum Umweltschutz zu gewinnen, analysiert man die vermutlichen Problemsituationen und Bedarfe aus der Perspektive der Bildungsanbieter im Sinne einer Erwartungs-Erwartungs-Verschränkung (Luhmann 1997) genauer. Dabei ist die Vermutung leitend, dass nicht moralische oder professionelle Gründe das handlungsleitende Motiv der Angebotsgestaltung darstellen, sondern individuelle und organisationale Wahrnehmungs- und Deutungsmuster der Unternehmen.

\section{7 Ökologische Bildung jenseits ethischer Kommunikation als Problemlösung und lebensweltbezogener Erkenntnisprozess}

Die Bildungsgegenstände für Organisationen finden ihren Ausdruck in einer Akteurs-Objekt-Relation und einer aktiv darauf bezogenen Gestaltung. Ein wichtiger Bezugspunkt nachhaltiger Entwicklung kann im Aspekt der Systemumwelt gesehen werden, worin Fragestellungen der Entwicklung von Technologie eingeschlossen sind. Ein zentrales Anliegen der analysierten Bildungsprogramme scheint es zu sein, zur Informationsgewinnung für die Unternehmen beizutragen.

Das Ziel der Informationsgewinnung kann aus wirtschaftsethischer Perspektive im weitesten Sinne in der Entwicklung einer Corporate-Stakeholder-Strategie gesehen werden. Hier wird dem Aspekt der Dominanz sozialer Kontexte für das unternehmerische Handeln Rechnung getragen, die diesen Aspekt einen starken „legitimativen“ Charakter gegenüber Stakeholdern beimessen, wie das Beispiel der CSR Initiative Rheinland verdeutlichen soll:

„CSR Strategien entwickeln bedeutet Wettbewerbsvorteile finden und nutzen" (CSR Initiative Rheinland) [1].

Eine Kombination aus Leitbildentwicklung, Nachhaltigkeitscheck und der Nachhaltigkeitsberichterstattung steht für eine Akzeptanz des wirtschaftlichen Handelns (Trifolium). Dabei geht es insbesondere um Compliance zum sicheren Umgang mit rechtlichen und gesellschaftlichen Anforderungen (IHK Nürnberg). Dazu wird ein strategisches und prozesshaftes Denken thematisiert (evoco), bei dem Nachhaltigkeit als möglichst ganzheitlicher unternehmerischer Ansatz und Managementprozess durch den/die Nachhaltigkeits-Manager/in implementiert wird (INUR). Insgesamt erscheint das Thema eher als Nachhaltigkeits-Marketing (CSR Beraternetz), auch wenn nachhaltiges Wirtschaften und CSR nicht als Marketinginstrumente gelabelt werden (sba-Initiative) beziehungsweise versucht wird, diesen Eindruck zu vermeiden.

Es können zwei unterschiedliche Arten von Aufklärungsund Informationsbedarfen unterschieden werden. Um die Corporate-Stakeholder-Strategie entwickeln zu können, benötigt ein Unternehmen zunächst Informationen über sich selbst. Zu den Informationen der Organisation über sich selbst gehören beispielsweise Methoden der unternehmensnahen Markt- und Umfeldanalyse (IMUG Beratungsgesellschaft), der Erstellung der eigenen Ökobilanz und des eigenen Carbon Footprints für die Nachhaltigkeitsbewertung (TÜV Rheinland). Die Informationen über sich selbst können in Form der Selbstanalyse durch unternehmensinterne Energie- und Umweltbeauftragte (TÜV Rheinland) ermittelt werden. In solchen Seminaren erlernen die Teilnehmenden den Umgang mit „[...] Elemente[n] des modernen Energiemanagements und [...] [der] Steigerung der Energieeffizienz durch systematische Vorgehensweisen bei der Aufdeckung von Einsparpotenzialen und der Umsetzung von Energieeinsparmaßnahmen“ (UI).

Die Generierung entsprechender Informationen stellt für die Unternehmenspolitik eine wichtige Säule dar, da diese Informationen sich als Klimaschutzaktivitäten für die Öffentlichkeitsarbeit und das Marketing nutzen und vermarkten lassen (UI). Die Ermittlung geeigneter Informationen kann auch an externe Dienstleister abgegeben werden, die individuelle Energie-Kennzahlen (Energy Performance Indicator (EnPI)) für ein Unternehmen ermitteln und für die unternehmerische Umweltpolitik aufbereiten (envidatec).

Die zweite Ebene des Informationsbedarfes bezieht sich auf die Umwelt des Unternehmens, wobei im Schwerpunkt auf die 
soziale Umwelt (gegenüber der natürlichen Umwelt) rekurriert wird. Hier lassen sich fünf Informationsfelder identifizieren, die für einen Umgang mit unvollständigen und überkomplexen Informationen notwendig erscheinen:

- Konzeptwissen, zum Beispiel Umweltmanagementsystem (UMS), Corporate Social Responsibility (CSR): Ein wichtiges Seminarthema sind die Grundlagen des Energie- und Umweltmanagements mit Arten der Managementsysteme, ihr Aufbau und die dazugehörigen Inhalte (kenic).

- Marktwissen, zum Beispiel Energiemarkt: Der Informationsbedarf umfasst unter anderem das „Erkennen und Verstehen des Zusammenspiels von Energieerzeugung (Art und Weise) und Energieverwendung (was, wo und in welcher Menge)“ (REFA). Dazu gehören auch Themen der „Energiesteuer, Stromsteuer, EEG, EEWärmeG, EnEV, Gesetze zur Kraft-Wärme-Kopplung, $\mathrm{CO}_{2}$-Emissionshandel, Förderprogramme, Wirtschaftlichkeitsberechnungen, Contracting“ (taw).

- Wirtschaftswissen, zum Beispiel Stakeholder: Aus ökonomischer Perspektive ist das Kosteneinsparpotenzial von Interesse (taw). Dazu gehören aber auch Informationen über wichtige Förderprogramme, Unterstützungsmöglichkeiten durch die interne Kostenrechnung, die Berechnung der Wirtschaftlichkeit von Investitionen und energetischen Maßnahmen sowie die Kenntnis von Finanzierungsmöglichkeiten (proterra).

- Rechtliches Wissen, zum Beispiel Energierecht: Rechtsgrundlagen bestehen aus einzuhaltenden Richtlinien, welche dokumentiert werden müssen (IHK Nordschwarzwald). In diesem Bereich geht es um das Kennen und Einhalten relevanter Haftungsregelungen (DGQ Umweltmanagement).

- Technologisches Wissen, zum Beispiel Energieeffizienz: Ein wichtiges Element stellen hier die Grundlagen der Energietechnologie wie der Wind-, Sonnen-, Bio- und Wasserenergie und anderen als Energiebereitstellungssysteme dar (btc). Bedeutsam ist aber auch die Auseinandersetzung mit Wirkungsbegriffen, der Umwandlung und Wertigkeit von Energie sowie energiewirtschaftlichen Begriffen zur Energieeinsparung (taw) Mit Bezug auf die Einführung von Umweltund Energie-Managementsystemen scheint ein erhöhter Bedarf an „Transparenz über die wesentlichen Energieströme der einzelnen Bereiche der Organisation“ (envidatec) zu bestehen.

Mithilfe der dargestellten Informationsfelder können zwei bildungstheoretische Konsequenzen für ein Begriffsverständnis ökologischer Bildung von und in Organisationen formuliert werden. Innerhalb der Programme wird sichtbar, dass die Bildungsprogramme einen Beitrag zur Lösung des ökonomischen Effizienzproblems beschreiben. Dieses Effizienzproblem beschreibt den zentralen Bezugspunkt der organisationalen Wirklichkeitskonstruktion. Mit Blick auf die Informationsfelder kann organisationspädagogisch in diesem Sinne die Relation einer „Bildung als Problemlösung“ (Thompson et al.
2014) und einer Bildung „[...] als lebensweltbezogenen Erkenntnisprozess“ (Schmitz 1984) hergestellt werden.

Mit diesen beiden Relationen wird ein nachhaltigkeitsorientiertes Lernen von seiner pädagogischen und seiner organisatorischen Seite als Kulturaufgabe greifbar. Nur eine wechselseitige Bezogenheit aus Lebensweltbezug und Problemlösung kann zu einer Förderung von Umweltlernprozessen führen. Mit ihr bietet sich eine Synthese aus Umweltbildung und Managementsystemen. Im Sinne einer bildungstheoretischen Perspektive ist das Verständnis individueller, sozialer und organisationaler Lernprozesse zu schärfen (Möller 2000).

\section{Fazit: Gestaltung einer Dialog- und Prozesskultur ökologischer Transformation}

Wirtschaftliches Handeln von Organisationen folgt aus ethischer Sicht kollektiv entwickelten „Angemessenheitsregeln“ (Hasse et al. 1996). Externe und interne institutionelle Umwelten der Organisationen sind dafür der Bezugsrahmen. Diese Umwelten bestehen aus Regeln, Normen und Werten, die dazu dienen, organisationales Handeln zu legitimieren. Legitimation ist ein zentraler Begriff im organisationssoziologischen Neo-Institutionalismus, der auf die Übereinstimmung von Erwartungen der Anspruchsgruppen und die Erwartungserfüllung durch die Organisation und innerhalb der Organisation abzielt (Hellmann 2006). In diesem Sinne der Erwartungsregulierung kann man für eine ökologische Bildung im Kontext der Organisationen aus wirtschaftsethischer Perspektive als wesentliches Element bildungstheoretisch eine makround eine mikroinstitutionalistische Perspektive unterscheiden. Sie repräsentiert einen Shift von einer moralischen Verantwortung zu einer strukturellen Verantwortung, wobei der Organisationspädagogik die maßgebliche Aufgabe zukommen kann, den Institutionalisierungsbedarf im Rahmen einer Prozessund Dialogkultur mikropolitisch auszugestalten.

- In der makroinstitutionellen Perspektive werden Erwartungen aus der Umwelt adaptiert, indem organisationale Strukturen und Verhaltensweisen homogenisiert werden (z. B. Schaffung der Position des Umweltschutzbeauftragten). Es wirkt hier ein Anpassungsdruck, der sich aus einer erforderlichen Legitimationszuschreibung ergibt. Diese Legitimationszuschreibung erfolgt seitens der Gesellschaft in Form von Recht und Umweltpolitik sowie auch von Seite der Kund/innen als zentraler Marktzugang. Die Folge ist eine Prozessethik, die meist über die Implementierung von Energie- und Umweltmanagementsystemen ihren Ausdruck findet.

- Diese Prozessethik sollte idealerweise von einer Dialogkultur als mikropolitischem Gestaltungsansatz begleitet werden, um den Prozess der Mikroinstitutionalisierung zu unterstützen. Dieser fragt nach der Rolle der Akteure innerhalb der Organisation (Führungskräfte oder Mitarbeitende) und danach, wie Verhaltensweisen als Regeln, Normen und Abläufe zu Institutionen führen (Süß 2009). 


\section{Anmerkung}

[1] Zitation des analysierten Programms: Archivnummer, Name Bildungsanbieter, Zeile (in MAXQDA)

\section{Literatur}

Arnold, R./Siebert, H. (1995): Konstruktivistische Erwachsenenbildung. Von der Deutung zur Konstruktion der Wirklichkeit. Baltmannsweiler, Schneider.

Arlt, P. D./Zech, P.D. (2015): Arbeit und Muße. Wiesbaden, Springer.

Beschorner, T./Behrens, T./Hoffmann, E./Lindenthal, A./Hage, M./Thierfelder, B./Siebenhühner, B. (2005): Institutionalisierung von Nachhaltigkeit. Eine vergleichende Untersuchung der organisationalen Bedürfnisfelder Bauen \& Wohnen, Mobilität und Information \& Kommunikation. Marburg, Metropolis.

Bolscho, D. (2010): Umweltkommunikation und Erziehung. In: Büscher, C.\&Japp, K. P. (Hrsg.) (2010): Ökologische Aufklärung: 25 Jahre „Ökologische Kommunikation“. Wiesbaden, VS Verlag für Sozialwissenschaften. 203-228.

Conrad, J. (2000): Nachhaltige Entwicklung. Einige begriffliche Präzisierungen oder der heroische Versuch einen Pudding an die Wand zu nageln. FFU-Report 07-2000. Berlin, Freie Universität Berlin.

de Haan, G. (2008): Gestaltungskompetenz als Kompetenzkonzept der Bildung für nachhaltige Entwicklung. In: Bormann, I./de Haan, G. (Hrsg.): Kompetenzen der Bildung für nachhaltige Entwicklung. Operationalisierung, Messung, Rahmenbedingungen, Befunde. Wiesbaden, VS Verlag für Sozialwissenschaften. 23-44.

Eblinghaus, H./Stickler, A. (1998): Nachhaltigkeit und Macht. Zur Kritik von Sustainable Development, 3. Aufl. Frankfurt am Main, IKO.

Engels, J. I. (2006): Naturpolitik in der Bundesrepublik: Ideenwelt und politische Verhaltensstile in Naturschutz und Umweltbewegung 1950-1980. Paderborn, Schöningh.

Eppler, E. (2011): Eine solidarische Leistungsgesellschaft: Epochenwechsel nach der Blamage der Marktradikalen. Bonn, Dietz.

Hasse, R./Krücken, G. (2005): Neo-Institutionalismus, 2. Aufl. Bielefeld, Transcript.

Hauff, V. (1991): Soziale und ökologische Verantwortung von Unternehmen. In: Dierkes, M./Zimmermann, K. (Hrsg.): Ethik und Geschäft: Dimensionen und Grenzen unternehmerischer Verantwortung. Wiesbaden, VS Verlag für Sozialwissenschaften. 73-86.

Hellmann, K.-U. (2006): Organisationslegitimität im Neo-Institutionalismus. In: Senge, K./Hellmann, K.-U. (Hrsg.): Einführung in den Neo-Institutionalismus. Wiesbaden, VS Verlag. 75-88.

Hengsbach, F. (1991): Wirtschaftsethik. Freiburg: Herder-Spektrum.

Homann, K. (1995): Ethik und Ökonomik. In: Kappler, E./Scheytt, T. (Hrsg.): Unternehmensführung - Wirtschaftsethik - gesellschaftliche Verantwortung. Gütersloh, WBV. 177-200.

Huber, J. (2011): Allgemeine Umweltsoziologie, 2. Aufl. Wiesbaden, VS Verlag für Sozialwissenschaften.

Karmasin, M. (1996): Ethik als Gewinn. Wien, Linde.

Krainer, L./Heintel, P. (2010): Prozessethik. Zur Organisation ethischer Entscheidungsprozesse. Wiesbaden, Verlag für Sozialwissenschaften.

Lederle, S. (2008): Die Ökonomisierung des Anderen. Eine neoinsitutionalistisch inspirierte Analyse des Diversity Management-Diskurses. Wiesbaden, VS Verlag für Sozialwissenschaften.

Luhmann, N. (2002): Das Erziehungssystem der Gesellschaft. Frankfurt am Main, Suhrkamp.

Luhmann, N. (1997): Die Gesellschaft der Gesellschaft. Bd. 1 und 2. Frankfurt am Main, Suhrkamp.

Luhmann, N. (1990): Die gesellschaftliche Moral und ihre ethische Reflexion. In: Ethik und Unterricht 1990/3: 4-14.

Luhmann, N. (1989): Ethik als Reflexionstheorie der Moral. In: Luhmann, N. (Hrsg.): Gesellschaftsstruktur und Semantik, Bd. 3. Frankfurt am Main: Suhrkamp. 358-447.

Luhmann, N. (1988): Wirtschaft der Gesellschaft. Frankfurt am Main: Suhrkamp.
Luhmann, N./Schorr, H. E. (1988): Strukturelle Bedingungen von Reformpädagogik. Soziologische Analysen der Moderne. In: Zeitschrift für Pädagogik 34/4: 463-480.

Möller, C. (2000): Umweltlernprozesse in Unternehmen. Münster, Waxmann.

Müller-Christ, G. (2008): Frames und Widerspruchsmanagement: Voraussetzungen für einen Wandel in Richtung nachhaltige Unternehmen. In: Lange, H. (Hrsg.): Nachhaltigkeit als radikaler Wandel. Die Quadratur des Kreises. Wiesbaden, VS Verlag für Sozialwissenschaften. 215-235.

Nethöfel, W. (1997): Unternehmensethik. Orientierung in der Krise. In: Geißler, H. (Hrsg.): Unternehmensethik, Managementverantwortung und Weiterbildung. Neuwie, Luchterhand. 106-118.

Proske, M. (2002): Pädagogisierung und Systembildung: Das Pädagogische im gesellschaftlichen Umgang mit dem Dritte-Welt-Problem. In: Zeitschrift für Erziehungswissenschaft 5/2: 279-298.

Radtke, F.-O. (1995): Interkulturelle Erziehung. Über die Gefahren eines pädagogisch halbierten Anti-Rassismus. In: Zeitschrift für Pädagogik 41/6: 853-864.

Schmitz, E. (1984): Erwachsenenbildung als lebensweltbezogener Erkenntnisprozess. In: Schmitz, E./Tietgens, H. (Hrsg.): Enzyklopädie Erziehungswissenschaft, Bd. 11: Erwachsenenbildung. Stuttgart, Klett-Cotta. 95-123.

Schüßler, I. (2007): Nachhaltigkeit in der Weiterbildung: theoretische und empirische Analysen zum nachhaltigen Lernen von Erwachsenen. Baltmannsweiler, Schneider Hohengehren.

Steinmann, H./Gerhard, B. (1992): Effizienz und Ethik in der Unternehmensführung. In: Homann, K. (Hrsg.): Aktuelle Probleme der Wirtschaftsethik. Berlin: Duncker und Humblot. 159-182.

Süß, S. (2009): Die Institutionalisierung von Managementkonzepten. Eine strukturationstheoretisch-mikropolitische Perspektive. In: Zeitschrift für Betriebswirtschaft 79/2, 187-212.

Tietgens, H. (1990): Erwartungen an Erwachsenenbildungsforschung. In: Kade, J. (Hrsg.): Fortgänge der Erwachsenenbildungswissenschaft. Frankfurt am Main, PAS/DVV. 56-63.

Thompson, C./Jergus, K. (2014): Zwischenraum Kultur „Bildung“ aus kulturwissenschaftlicher Sicht. In: von Rosenberg, F./Geimer, A. (Hrsg.): Bildung unter Bedingungen kultureller Pluralität. Wiesbaden, Springer. 9-26.

Towers, J./Kohler, M. (2008): Ökologie und Design. In: Erlhoff, M./Marshall, E. (Hrsg.). Board of International Research in Design. Basel, Birkhäuser Verlag. 297-299.

Treml, A. K. (2000): Allgemeine Pädagogik. Grundlagen, Handlungsfelder und Perspektiven der Erziehung. Stuttgart, Kohlhammer.

Wieland, J. (1991): Die Ethik der Wirtschaft als Problem lokaler und konstitutioneller Gerechtigkeit. In: Wieland, J. (Hrsg.): Wirtschaftsethik und Theorie der Gesellschaft. Frankfurt am Main: Suhrkamp. 7-31.

\section{AUTOR + KONTAKT}

Dr. Thomas Prescher ist Professor für Berufspädagogik an der Wilhelm Löhe Hochschule für angewandte Wissenschaften.

Wilhelm Löhe Hochschule für angewandte Wissenschaften, Merkurstr. 41, 90763 Fürth. Tel.: +49 91172301520 ,

E-Mail: thomas.prescher@sowi.uni-kl.de, Website: https://www.wlh-fuerth.de/ gesundheitshochschule/

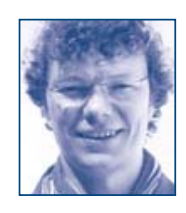

